\title{
Anton ALLAHAR, Caribbean Charisma: Reflections on Leadership, Legitimacy, and Populist Politics
}

Sylvie Calixte

\section{(2) OpenEdition}

Édition électronique

URL : http://journals.openedition.org/plc/265

DOI : $10.4000 /$ plc. 265

ISSN : 2117-5209

Éditeur

L'Harmattan

\section{Édition imprimée}

Date de publication : 14 janvier 2004

Pagination : 161-167

ISBN : 2-7475-7061-4

ISSN : 1279-8657

\section{Référence électronique}

Sylvie Calixte, «Anton ALLAHAR, Caribbean Charisma: Reflections on Leadership, Legitimacy, and Populist Politics », Pouvoirs dans la Caraïbe [En ligne], 14 | 2004, mis en ligne le 14 mars 2011, consulté le 22

septembre 2020. URL : http://journals.openedition.org/plc/265 ; DOI : https://doi.org/10.4000/plc.265 


\section{NOTES DE LECTURE}

\section{Caribbean Charisma: Reflections on Leadership, Legitimacy, AND POPUlist Politics}

EDITED BY ANTON ALLAHAR - 2001

par Sylvie CALIXTE

doctorante en droit

Cet ouvrage a pour objet les fondements du leadership politique dans les Caraïbes, en analysant le succès de sept hommes d'Etat caribéens depuis les premiers jours de la décolonisation.

Région de contrastes et de similitudes, la Caraïbe est connue à la fois pour le mythe de sa richesse et pour la richesse de ses mythes. Paradis touristiques et tempêtes tropicales, paradis fiscaux et pauvreté endémique, noirs secouant les chaînes coloniales, et blancs craignant pour leur sécurité. Fonction du point de vue adopté, la Caraỉbe peut représenter un tout, défini par une continuité culturelle, une similitude raciale, et une stabilité politique; ou bien, un complexe d'entités spécifiques, mélanges de cultures hétéroclites, confrontées aux conflits raciaux et ethniques, aux particularismes nationaux et linguistiques, et sans véritable unité politique. Entre unité et division, la question oppose ceux qui parlent d'un processus d'unité caribéenne, à ceux qui voient l'indifférence des peuples de la Caraïbe les uns envers les autres. Mais quelles que soient ces controverses, il est avéré que de nombreuses personnalités de réputation mondiale sont issues de cette région : Prix Nobel, artistes et athlètes de haut niveau; au plan politique, les leaders caribéens, auxquels cet ouvrage est consacré, ont acquis depuis longtemps une envergure internationale de chefs d'Etat. Il s'agit ici d'analyser les succès politiques de plusieurs leaders caribéens en 
remontant au début de la décolonisation politique des années 1960, à une époque où, sous la promesse d'une libération de la tutelle coloniale, le climat politique des Caraỉbes était empreint des prémices d'une euphorie nationale.

Saisissant le moment propice du courant politique, ces leaders, Errol Barrow (Barbade), Fidel Castro (Cuba), Maurice Bishop et Eric Gairy (Grenade), Forbes Burnham et Cheddi Jagan (Guyana), Michael Manley (Jamaïque), et Eric Williams (Trinidad), à des degrés variables et dans leur style unique, ont exploité leur charisme naturel, qu'ils ont utilisé pour mettre en œuvre des politiques populistes. Ils ont joué sur des idées comme l'autodétermination dans l'optique de gagner et de cimenter la loyauté de leurs partisans. Par d'habiles manipulations des identités sociales et politiques, s'appuyant sur le populisme ou le nationalisme, sur les oppositions de classes, et même de races, ils ont recueilli un soutien inconditionnel des différentes couches des populations locales. Ce fut une période de forte mobilisation politique contre le maître colonial qui a été rebaptisé «L'Autre ».

Ce livre est un collectif, composé de huit chapitres, dans lesquels huit chercheurs différents tentent de mettre en évidence le niveau auquel le concept de charisme relève d'une appréciation des triomphes politiques ou des succès des leaders en question. En procédant d'une analyse critique des bases de la légitimité politique de Max Weber, l'ouvrage examine comment des leaders modernes ont été capables d'utiliser leur charisme naturel dans la poursuite d'une finalité politique spécifique. S'ensuit alors, un ensemble de réflexions sur les thèmes du charisme et du populisme à travers la biographie politique et intellectuelle des leaders caribéens choisis, et de l'incidence de ces thèmes sur la pratique politique des différents pays des Caraỉbes.

En premier lieu, les considérations générales et théoriques d'Anton Allahar portent sur le lien entre leadership, charisme et politique dans la Caraibe, sans se limiter, toutefois, aux visées sociologiques de Max Weber, car développées dans un contexte social et politique très éloigné de la Caraïbe. Pour autant, le concept de charisme est ici pris en compte dans la mise en évidence du niveau auquel il intervient pour 
expliquer la dynamique de la psychologie politique entre les leaders et leurs partisans.

C'est dans un processus de crise aiguë et de changements, de migrations politiques majeures qu'ont émergé ces leaders politiques exceptionnels, arrivés au pouvoir grâce à la démocratie libérale, à l'exception toutefois de Fidel Castro et Maurice Bishop. Dans le langage de Weber, il s'est agi de leaders modernes dont l'autorité et la légitimité résidaient dans une " séduction rationnelle " à l'égard de leurs partisans, et dans le fait que leur leadership reposait sur des bases légales. Mais alors comment articuler ce leadership avec le populisme ? Et en dehors du contexte de la démocratie libérale, comment le charisme et le populisme pourraient-ils expliquer les succès de Castro et Bishop? Finalement, en quoi ces considérations servent-elles à enrichir les théories courantes du leadership politique?

A la Barbade Hilbourne Watson explique qu'Errol Barrow eut recours à la rhétorique pour contraindre la classe moyenne à acquérir le pouvoir, mais sans aucun plan permettant corrélativement une limitation effective du pouvoir déjà massif de la domination économique de la «minorité des trois pour cent ». En effet, cette majorité n'étant pas de « culture capitaliste », elle ne pouvait mettre en œuvre ce pouvoir au-delà d'abstractions universelles. Liberté et justice supportent alors la trace de la force parce que la version de la justice qui prévaut dans des sociétés comme Barbade est ancrée dans la force. Et le charisme ne s'élève jamais au-dessus des contradictions de classes, de genre, ou d'ethnies ; ou alors il déplace ce qu'il y a de plus fondamental dans ces contradictions. La société politique, incluant la société civile, avec ses complexes issus des institutions, joue des rôles dans lesquels significations et valeurs sont produites, et dans lesquelles les différentes classes sociales servent à légitimer le statu quo à travers leur consentement spontané à être régies.

Pour Grenade, Pedro Noguera se demande quelles sont les réponses populaires au leadership charismatique des deux leaders, Eric Gairy en 1950 et Maurice Bishop au début des années 1970. Max Weber a soutenu que le support d'un leader charismatique est basé sur des réponses populaires irrationnelles et émotionnelles, le leader étant perçu 
comme «possédant des dons et des talents extraordinaires ». La méthode de l'enquête de données, est utilisée ici pour analyser les réponses populaires aux qualités charismatiques de Gairy et Bishop, dans un examen critique de ce qui soutient un leader charismatique et qui est enraciné dans les réponses irrationnelles du peuple à une souffrance sociale. En effet l'émergence de ces deux leaders coïncide avec les bouleversements sociaux majeurs qui ont apporté des changements politiques significatifs pour les pauvres et les travailleurs de l'île.

Pour Linden Lewis, le charisme post-colonial de Forbes Burnham, un des deux leaders du Guyana, est un paradoxe basé sur une rhétorique flamboyante, un charme paysan, le culte de la personnalité, et sa préoccupation de laisser sa place dans l'histoire. Lewis examine son émergence en tant que leader politique charismatique, et la consolidation de son pouvoir depuis le colonialisme, à travers l'indépendance, jusqu'à la phase de la République socialiste coopérative. Pendant son évolution politique, Burnham s'est métamorphosé d'une figure populaire, de politicien indigène, en un leader incontestable, dont le leadership, bien que décidément autocratique, continue de jouir d'un certain élan populaire, et s'est étendu au-delà de son Guyana natal et des Caraïbes. Champion des causes politiques du tiers-monde tel que le Nouvel Ordre Economique International, il camouflera ainsi sa politique autoritaire intérieure, n'hésitant pas à exploiter, politiquement, la question des races dans une région socialement polarisée par une histoire de conflits raciaux.

Percy Hintzen présente l'autre leader du Guyana : Cheldi Jagan et son attirance pour le charisme ou plutôt, son incarnation des aspirations du prolétariat et de la pesanteur de la créolité. Jagan représentait une alternative aux attributions raciales du colonialisme. Il navigua entre deux pôles opposés. Ses racines l'ancraient dans le monde rural, mais il était poussé vers un monde chrétien de l'ouest composé d'élites non prolétaires et non paysannes. Il avait également une répulsion pour le snobisme de l'élite créole et pour l'exclusivité des représentations et des pratiques du monde des Antilles, mais ce monde se révélait particulièrement attirant, contrariant la prétendue supériorité «des 
plaisirs simples de la campagne [...] pleins d'expériences riches ». Ces contrastes ont façonné la quête de toute sa vie à mettre en phase l'éthique égalitaire et libérale de l'antillanité formulée en langage de liberté, tout en cherchant à échapper à l'exploitation et au snobisme qu'elle engendrait. Telles sont les contradictions ayant généré les institutions qui ont défié le cœur du capitalisme racialisé antillais. C'est dans cette institutionnalisation que résida la charisme de Jagan.

Pour la Trinidad d'Eric Williams, Patricia Mohammed se demande quelles visions et craintes avait Eric Williams pour une société qu'il a dirigé pendant trente ans. Quelle connaissance et perspicacité politique pouvait-il avoir acquis pour avoir entraîné avec succès son pays dans une révolte anti-coloniale, et nourri son peuple avec le cadeau du premier livre écrit sur l'histoire locale à propos de la naissance de l'indépendance vis-à-vis de l'Angleterre? Williams a personnellement construit un support sur le long terme pour lui-même et pour le parti qu'il a conduit à la victoire, et dans ce processus il fit preuve d'une certaine hardiesse, inspira la loyauté des femmes, qu'il a amenées au bercail de la culture politique quand il était encore impopulaire d'agir ainsi. Maintenant, pour la majorité de la population, Williams demeure une énigme du premier au dernier jour de son règne et même au-delà de sa mort.

Pour la Jamaïque de Michael Manley, Brian Meeks tente de déconstruire «les composantes d'une carrière charismatique ». Meeks considère que Michael Manley est un des hommes politiques les plus importants de la période post-indépendantiste, se demandant ce qui a contribué à son accession au pouvoir et à son écrasante et charismatique personnalité. Quel est son héritage naturel en tant que fils de Norman Manley, un des «pères » reconnus de l'Etat jamaïcain ? Comment a-t-il montré la voie vers l'avant en tant que militant et leader syndical affirmé ? Quel a été le rôle de la philosophie millénaire dans la transformation du trop humain Michael en une demi divinité ? Et quels furent les effets de race et de classe dans cette transition remarquable? Ceci et d'autres question sont débattues sur fond de théorie webérienne 
des notions de leadership charismatique et subséquemment de marxisme et néo-marxisme.

Enfin, dans le chapitre consacré à Cuba, Nelson Valdés s'attaque au charisme de Fidel Castro et au rôle qu'il a joué dans la construction et la direction de la révolution cubaine. Son système politique est le reflet d'un équilibre difficile entre des institutions formelles et une autorité charismatique. Celle-ci fonctionne sur les qualités personnelles de Castro, de même que sur celles qui lui ont été attribuées par le peuple cubain. Le leadership de Castro est basé sur une réaffirmation permanente de son autorité à travers la mobilisation de la population. Son contact avec la population est un véritable droit au pouvoir, et ce droit, qu'il a gagné comme guérillero, a été puissamment soutenu par le peuple qui le reconnaît comme quelqu'un qui est béni des dieux. L'autorité charismatique est un travail de revendication. La légitimité de Castro ne peut être séparée des bienfaits que la population a reçus des redistributions politiques du régime. Il conduisit le processus de mise en place de nouvelles institutions ; introduisit la méthode de la mobilisation de masse ; il définit aussi le modèle de concentration des ressources en dehors des zones urbaines, et a transmis au peuple la croyance qu'il a un droit inné au travail, à la protection de la santé et à l'éducation.

Puisant dans les méthodes d'analyse de la science politique, de l'histoire et des sciences humaines en général, cet ouvrage veut en finir avec l'imagerie d'Epinal des Antillais érigée par l'Occident.

Flamboyants, effervescents et séduisants, certes, subversifs et arrogants sans aucun doute, mais aussi modernes, voyageurs, cosmopolites et créatifs, ce livre montre que les habitants des Caraibes disposent d'un haut degré de culture et de conscience politique.

Du premier contact à la décolonisation en passant par l'esclavage et la dépendance économique, les Antillais, pris dans le champ de la communauté mondiale, ont su intégrer la culture de l'Autre tout en se constituant une position subjective spécifique. 
Même si les régions de la Caraïbe sont examinées ici par des auteurs différents, usant de méthodes d'analyse différentes, une diagonale se dessine qui met en évidence un vecteur commun à tous les peuples de la Caraỉbe, une forme de capacité à générer et à assumer leur destin politique. 